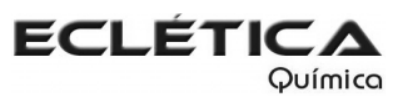

www.scielo.br/eq

www.ecletica.iq.unesp.br

Volume 33, número 4, 2008

\title{
Desenvolvimento e aplicação de método GC-MS/MS para análise simultânea de 17 HPAs em material particulado atmosférico
}

\author{
J. Cristale*, F. S. Silva, M. R. R. Marchi \\ Departamento de Química Analítica, Instituto de Química, Universidade Estadual Paulista, Rua. \\ Francisco Degni s/no - 14800-900, Araraquara, São Paulo, Brasil. \\ *joycecristale@iq.unesp.br
}

\begin{abstract}
Resumo: Os hidrocarbonetos policíclicos aromáticos (HPAs) estão associados ao aumento da incidência de diversos tipos de cânceres no homem. Essas moléculas são formadas principalmente na queima incompleta de matéria orgânica, sendo encontradas em todos os compartimentos ambientais. Órgãos regulamentadores das áreas ambiental e de saúde ocupacional consideram 17 HPAs como contaminantes atmosféricos prioritários. Este trabalho apresenta um método para análise simultânea destes HPAs utilizando-se a cromatografia a gás acoplada à espectrometria de massas operando no modo tandem (GC-MS/MS). Os limites de detecção e quantificação do método mostraram-se até 5 vezes inferiores aos obtidos no método GC-MS (SCAN). O método mostrou-se seletivo para análise de HPAs em extratos de amostras de material particulado atmosférico. Uma análise comparativa de dois sistemas de solventes (diclorometano/metanol 4:1 v/v e hexano/acetona 1:1 v/v) para a extração de HPAs, utilizando amostras de material particulado atmosférico, revelou que ambas as misturas de solventes possuem poder de extração semelhante. Os resultados sugerem que é possível realizar extração de HPAs de material particulado atmosférico em ultra-som com a mistura hexano/acetona (1:1), que é menos tóxica em relação à mistura diclorometano/metanol (4:1), bastante utilizada nestas análises, sem perdas significativas na exatidão do método.
\end{abstract}

Palavras chave: hidrocarbonetos policíclicos aromáticos; GC-MS/MS; material particulado atmosférico.

\section{Introdução}

Os hidrocarbonetos policíclicos aromáticos (HPAs) compreendem uma família de compostos que possuem dois ou mais anéis aromáticos condensados. Estas substâncias, têm ampla distribuição e são encontradas como constituintes de misturas complexas em todos os compartimentos ambientais[1, 2]. Os HPAs podem ser encontrados em plantas terrestres e aquáticas, solos, sedimentos, águas continentais e marinhas e na atmosfera[3, 4, 5, 6, 7, 8, 9, 10, 11]. Dezesseis destes compostos estão incluídos como poluentes prioritários pela Agência Norteamericana de Proteção Ambiental - EPA[12], enquanto o Instituto Nacional de Saúde e Segurança Ocupacional, também dos EUA, NIOSH[13] prioriza dezessete HPAs para investigação. O composto adicional na lista NIOSH é o benzo[e]pireno. A Figura 1 apresenta as estruturas dos 17 HPAs prioritários segundo o NIOSH. 
Os HPAs são formados em processos de combustão incompleta a altas temperaturas, e deste modo, são essencialmente emitidos por todos os tipos de combustão. Quando a matéria orgânica é queimada, em geral é formada uma grande variedade de HPAs em diferentes níveis de concentração, e a complexidade das misturas de HPAs dependem das fontes emissoras [14]. Dentre suas inúmeras fontes, podem ser citadas as de origem natural, como por exemplo, os incêndios florestais que ocorrem espontaneamente e as erupções vulcânicas; e as de origem antropogênica, tais como: processos de<smiles>c1cc2c3c(cccc3c1)CC2</smiles>

Acenafteno<smiles>c1ccc2cc3c(ccc4ccccc43)cc2c1</smiles>

Benzo[a]antraceno<smiles></smiles>

Benzo[g,h,i]perileno<smiles>c1ccc2c(c1)ccc1c3ccccc3ccc21</smiles>

Criseno<smiles>c1ccc2c(c1)Cc1ccccc1-2</smiles>

Fluoreno<smiles>O=C1c2ccccc2C2=CC=CC12</smiles><smiles></smiles>

Acenaftileno<smiles>C1=Cc2cc(cc3ccccc23)-c2ccccc21</smiles>

Benzo[e]acefenantrileno<smiles>c1ccc2c(c1)cc1ccc3cccc4ccc2c1c34</smiles>

Benzo[a]pireno

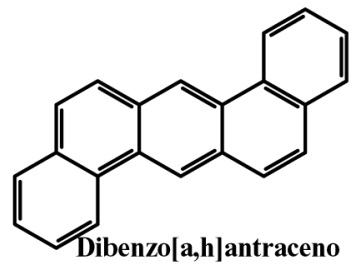<smiles>CC(C)(C)C1=CC2=CC=Cc3ccc4ccc2c1c4c3-c1ccccc1</smiles><smiles>c1ccc2cc3ccccc3cc2c1</smiles>

Antraceno<smiles>c1ccc2cc3c(cc2c1)-c1cccc2cccc-3c12</smiles>

Benzo[k]fluoranteno<smiles>c1ccc2c(c1)c1cccc3ccc4cccc2c4c31</smiles>

Benzo[e]pireno<smiles>c1ccc2c(c1)-c1cccc3cccc-2c13</smiles>

Fluoranteno<smiles>c1ccc2ccccc2c1</smiles>

Naftaleno<smiles>c1cc2ccc3cccc4ccc(c1)c2c34</smiles>

Pireno

Figura 1. Estruturas dos HPAs considerados prioritários pelo NIOSH[13] (NATIONAL INSTITUTE OF OCCUPATIONAL SAFETY AND HEALTH) 
combustão de material orgânico (particularmente a exaustão de motores a diesel ou a gasolina), queima de biomassa vegetal, exaustão de plantas de incineração de rejeitos, fumaça de cigarro e processos industriais, por exemplo, a produção de alumínio e a gaseificação do coque [2]. Os níveis de concentração de HPAs no ar atmosférico têm sido amplamente estudados, sobretudo em ambientes urbanos, que têm como principal fonte dessas moléculas a queima de combustível fóssil em motores a diesel e a gasolina, as atividades industriais e a queima de biomassa [15, 16, 17, 18]. Dessa maneira, diante dos riscos potenciais que a exposição humana a HPAs pode causar, métodos de análise que sejam capazes de detectar concentrações bastante baixas dessas moléculas são necessários a fim de possibilitar o monitoramento delas em todos os compartimentos que possam estar presentes.

As propriedades físicas e químicas das moléculas de HPAs permitem que elas sejam analisadas tanto por cromatografia gasosa (GC) $[4,10$, 19] quanto por cromatografia líquida de alta eficiência (HPLC)[16, 20, 21]. É importante ressaltar que na análise de traços são fundamentais métodos analíticos com baixos limites de detecção. O detector de espectrometria de massas tem sido muito utilizado na análise de HPAs em matrizes ambientais, por ser um detector que além de apresentar baixos limites de detecção, oferece informação estrutural dos compostos.

Uma ferramenta que alguns sistemas sofisticados de GC-MS oferecem é a análise no modo tandem (MS/MS). Nessa técnica, as moléculas de interesse sofrem fragmentação duas vezes. Primeiramente as moléculas são fragmentadas a 70 eV e então o sistema é capaz de isolar e refragmentar um fragmento selecionado (íon pai), gerando o espectro obtido do íon pai. Para escolha do íon pai deve ser realizada uma análise no modo GC-MS (SCAN), e a partir do espectro de massas da molécula de interesse, é selecionado o íon de maior intensidade para ser refragmentado. $\mathrm{O}$ equipamento utiliza uma ampla faixa de frequiência de onda para o isolamento do íon pai, que acontece em duas etapas: primeiramente são expulsos os íons de $\mathrm{m} / \mathrm{z}$ menor que o íon pai e em seguida os de $m / z$ maior que do íon pai[22]. A análise no modo tandem propicia maior seletividade e sensibilidade do sistema GC-MS.
Neste trabalho, foi desenvolvido método GC-MS/MS de análise de HPAs para aplicação a análise de material particulado atmosférico.

\section{Material e métodos}

\section{Reagentes e equipamentos}

Os padrões de HPAs sólidos individuais (pureza $>98 \%$ ) foram obtidos do laboratório Dr. EHRENSTORFER GMBH (Augsburg, Alemanha): naftaleno (naf), acenaftileno (aceftl), acenafteno (acft), fluoreno (fluo), fenantreno (fen), antraceno (ant), fluoranteno (flu), pireno (pir), benzo[a]antraceno (b[a]a), criseno (cri), benzo[e]acefenantrileno $(\mathrm{b}[\mathrm{e}] \mathrm{ac})$, benzo[a]pireno $(\mathrm{b}[\mathrm{a}] \mathrm{p})$, benzo[e]pireno (b[e]p), dibenzo[ah]antraceno (d[a,h]a), indeno(1,2,3-cd)pireno (ind); com exceção do benzo[k]fluoranteno $(b[k] f)$ e ben$\mathrm{zo}[\mathrm{g}, \mathrm{h}, \mathrm{i}]$ perileno (b[ghi]p) que foram fornecidos pela SIGMA-ALDRICH BRASIL Ltda. O isooctano grau HPLC, utilizado na preparação das soluções, foi fornecido pela MALLINCKRODT CHEMICALS.

As análises foram realizadas em um cromatógrafo a gás acoplado a um detector de espectrometria de massas, modelo GC 3800 MS Saturn 2000 com autosampler 8200, da marca VARIAN.

Desenvolvimento de método GC-MS (SCAN) de análise de 17 HPAs

Para o desenvolvimento do método cromatográfico GC-MS (SCAN) foram utilizadas soluções mistas de 17 HPAs em isooctano, preparadas a partir das soluções padrão individuais de cada HPA, cujas concentrações variaram de 1 a $0,001 \mu \mathrm{g} \mathrm{mL}^{-1}$. A solução mista de HPAs de concentração $1 \mu \mathrm{g} \mathrm{mL}^{-1}$ foi utilizada para otimização das condições cromatográficas. Diversas rampas de aquecimento foram utilizadas para encontrar as melhores condições de separação cromatográfica dos 17 compostos. As programações de temperatura utilizadas estão apresentadas na Tabela 1. O impacto eletrônico foi de $70 \mathrm{eV}$, e scan rate utilizado foi de 0,5 scan/seg. $\mathrm{O}$ gás de arraste utilizado foi hélio à uma pressão de $89 \mathrm{kPa}$ e vazão de $1 \mathrm{~mL} \mathrm{~min}^{-1}$. O volume de injeção foi de $2 \mu \mathrm{L}$, utilizando split na razão $1 / 5$. 
Desenvolvimento de método GC-MS/MS de análise de 17 HPAs

Os íons pai foram selecionados para cada HPA a partir do fragmentograma referente a cada pico obtido nas análises por GC-MS (SCAN). Foi realizado um estudo da influência da energia de excitação, utilizando as voltagens 25,50 e $75 \mathrm{~V}$ no modo não-ressonante e $0,20,0,5020,75 \mathrm{~V}$ no modo ressonante. Uma vez desenvolvido o método GC-MS/MS, foi construída uma curva analítica a partir de soluções mistas contendo padrões de 17 HPAs.

\section{Análise de amostras de material particulado} atmosférico

Nessa etapa do trabalho, foi realizado um estudo preliminar da eficiência de extração de HPAs em material particulado atmosférico utilizando duas misturas de solventes, diclorometano e metanol na proporção volumétrica 4:1 (DCM/MeOH 4:1 v/v) e uma solução isovolumétrica de hexano e acetona. Para isso, foram utilizadas três amostras de material particulado atmosférico. $\mathrm{O}$ material particulado foi coletado utilizando um amostrador Handi-vol (Energética, Brasil) e filtro de fibra de vidro (diâmetro de $5 \mathrm{~cm}$, Energética). As amostras foram coletadas na cidade de Araraquara. Outros detalhes da amostragem não são relevantes neste texto, uma vez que os filtros foram utilizados apenas para comparação entre os dois métodos de extração, que por sua vez foram aplicados em paralelo a cada amostra. Cada filtro foi cortado em duas partes iguais e cada parte foi submetida à extração com uma das misturas de solventes. A extração foi efetuada utilizando 3 porções de 10 $\mathrm{mL}$ de cada sistema de solvente em banho de ultra-som por $10 \mathrm{~min}$. Após a eliminação do solvente de extração (usando um leve fluxo de $\mathrm{N}_{2}$ ), o resíduo foi redissolvido em $200 \mathrm{~mL}$ de isooctano e analisado por CG-MS/MS.

\section{Resultados e discussão}

\section{Desenvolvimento de método GC-MS (SCAN)}

Diversas programações de temperatura (Tabela 1) foram testadas a fim de obter a melhor resolução possível na análise simultânea de 17 HPAs. A Figura 2 apresenta os cromatogramas obtidos em cada método utilizado. Pode ser observado em todos os cromatogramas que os

Table 1. Condições utilizadas no desenvolvimento do método GC-MS (SCAN).

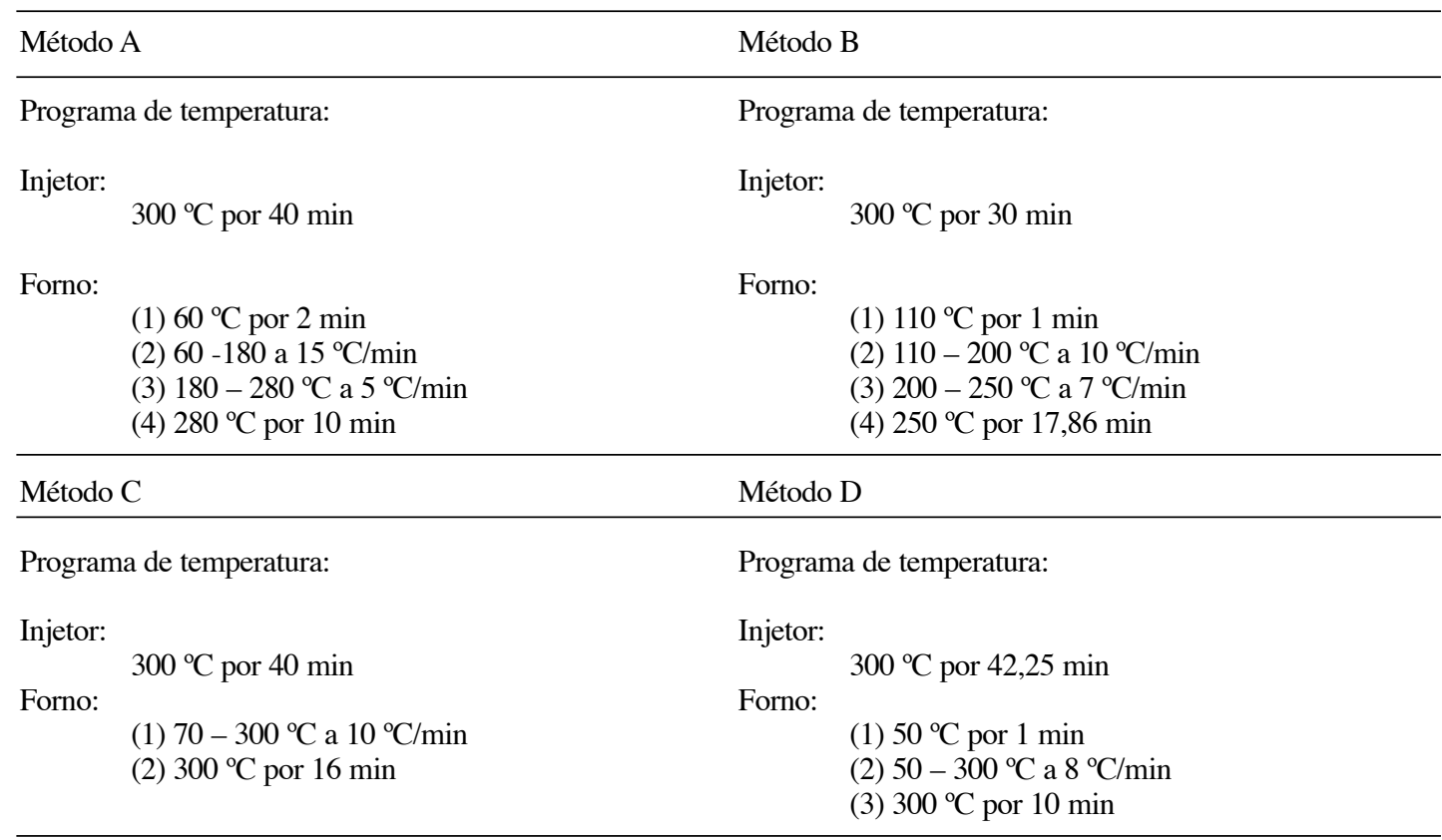


últimos picos, referentes às moléculas de HPAs de maiores ponto de ebulição, são alargados e sobrepostos, perdendo resolução. Isso ocorre porque essas moléculas levam um tempo relativamente longo para eluir da coluna, favorecendo os equilíbrios entre a fase móvel e a fase estacionária, aumentando o tempo de saída entre a primeira e a última molécula de uma substância que elui do sistema cromatográfico, causando o alargamento do pico. Esse comportamento pode ser evitado aumentando as condições de temperatura do sistema, porém isso resultaria em pior resolução das moléculas de HPA de menores P.E., causando sobreposição de picos e/ou coeluição, conforme verificado em testes preliminares.

Os Métodos A e D não permitem a identificação do indeno[1,2,3-c]pireno, diben-

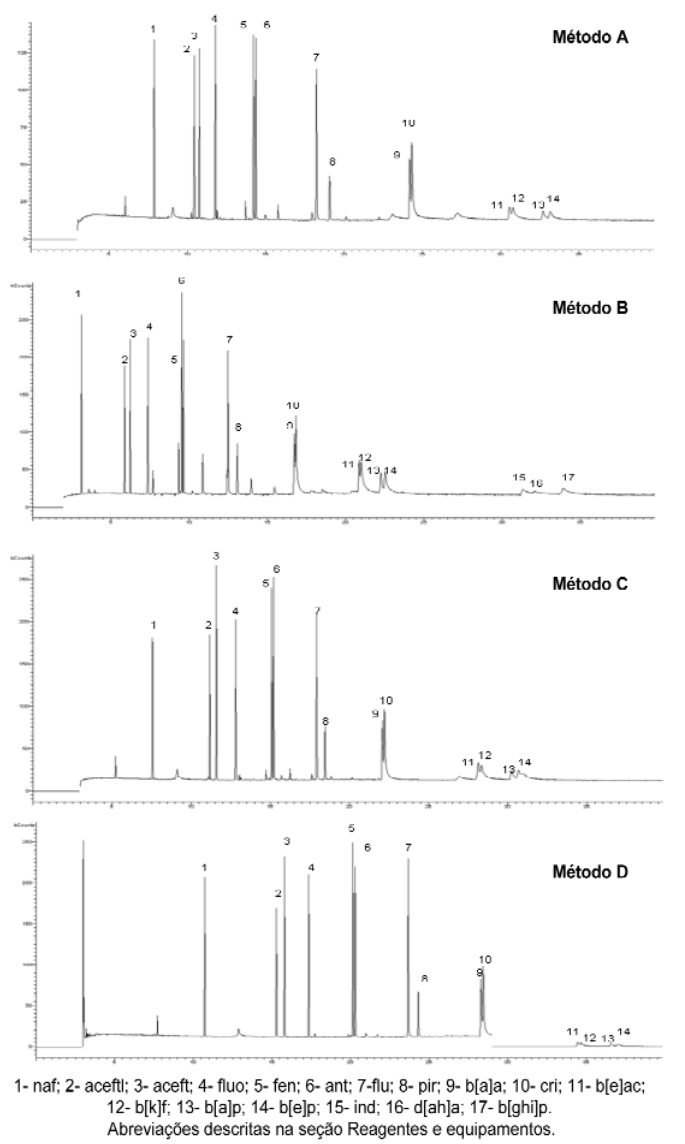

Figure 2. Cromatogramas referentes aos Métodos A, B, C e D, para análise simultânea de 17 HPAs. Está apresentado o trecho dos cromatogramas onde é possível a identificação de picos. $\mathrm{zo}[\mathrm{a}, \mathrm{h}]$ antraceno e benzo[g,h,i]perileno, pois os picos gerados por essas moléculas nesses métodos são tão alargados que não é possível distingui-los da linha de base. A análise dos fragmentogramas originados do Método $\mathrm{C}$ revelaram que essas mesmas moléculas coeluem com benzo[e]pireno, conforme pode ser observado na Figura 2, onde é formado um ombro no lado direito do pico indicado pelo número 14 no cromatograma referente ao Método $\mathrm{C}$. $\mathrm{O}$ método que permitiu a melhor separação dos 17 HPAs foi o Método B, que foi selecionado para o desenvolvimento do método GC-MS/MS.

\section{Desenvolvimento de método GC-MS/MS}

As condições de temperatura do Método B foram utilizadas para desenvolvimento do método GC-MS/MS. Os fragmentogramas referentes a cada HPA foram analisados para verificação do íon de maior intensidade (íon base), que foi selecionado para ser refragmentado. A Tabela 2 apresenta o íon pai para cada HPA.

No modo MS/MS de análise, os fragmentos originados a partir do íon pai (íons filhos) são formados a partir da colisão que induz a dissociação (CID - colision induced dissociation). A distribuição de $\mathrm{m} / \mathrm{z}$ dos íons resultantes do processo CID depende das características do íon pai e da quantidade de energia cinética translacional que será convertida em energia vibracional interna. A energia cinética translacional do íon pai pode ser aumentada utilizando-se dois modos de excitação: ressonante e não-ressonante. $\mathrm{O}$ modo não-ressonante $(0 \mathrm{a}$ 100V) é resultado da interação da espécie iônica com um campo elétrico que aumenta a ener-

Table 2. Íon pai correspondente a cada HPA.

\begin{tabular}{llll}
\hline HPA & $\mathrm{m} / \mathrm{z}$ HPA & $\mathrm{m} / \mathrm{z}$ \\
\hline Naftaleno & 128 & Criseno & 228 \\
Acenaftileno & 152 & Benzo[e]acefenantrileno & 253 \\
Acenafteno & 153 & Benzo[k]fluoranteno & 253 \\
Fluoreno & 165 & Benzo[a]pireno & 251 \\
Fenantreno & 178 & Benzo[e]pireno & 251 \\
Antraceno & 178 & Indeno[1,2,3-cd]pireno & 276 \\
Fluoranteno & 202 & Dibenzo[a,h]antraceno & 278 \\
Pireno & 202 & Benzo[g,h,i]perileno & 276 \\
Benzo[a]antraceno & 228 & \\
\hline
\end{tabular}


gia potencial do íon, aumentando sua energia translacional. Uma porção da energia cinética é convertida em energia translacional ao sofrer colisões no trap. No modo ressonante (0 a $1 \mathrm{~V})$ um campo elétrico oscilante aplicado é orientado ao longo do eixo axial do trap, dando origem a uma componente axial de movimento no íon que é ressonantemente excitada, causando aumento da energia interna do íon[22].

Dessa maneira, no desenvolvimento do método GC-MS/MS é necessário determinar a energia de excitação adequada para que ocorra a quebra do fragmento selecionado, e obter ganhos em limite de detecção da técnica. Para isso, foi realizado um estudo para determinar a melhor energia de excitação para as 17 moléculas de HPAs. Esse estudo foi realizado variando-se essa energia de excitação de 25,50 e $75 \mathrm{~V}$ no modo não-ressonante e $0,2,0,5$, e $0,75 \mathrm{~V}$ no modo ressonante. A determinação da melhor energia de excitação foi realizada através da análise comparativa da intensidade do sinal cromatográfico obtido referente a cada energia. O modo ressonante mostrou-se inadequado para análise dos HPAs, pois foram observadas perdas significativas em detectabilidade em comparação ao modo não-ressonante, que originou picos até dez vezes mais intensos. A energia de excitação de $25 \mathrm{~V}$ no modo não-ressonante foi a que permitiu boa resposta para todas as moléculas em estudo, e por isso foi escolhida para o método GC-MS/MS. A alta estabilidade dos íons pai, devido sua estrutura molecular, impossibilitou que sua fragmentação fosse significativa nas condições utilizadas. O íon filho é o próprio íon pai em todos os casos, obtendo-se um método cromatográfico mais seletivo que o GC-MS (SCAN).

Após selecionar a energia de excitação de $25 \mathrm{~V}$ não-ressonante, foram realizados testes a fim de melhorar a resolução dos 3 últimos picos, aumentando-se a taxa de aquecimento a partir de $23 \mathrm{~min}$. A nova rampa de aquecimento testada (Método E) foi: $100^{\circ} \mathrm{C} \quad(1 \mathrm{~min})-100^{\circ} \mathrm{C}-$ $10^{\circ} \mathrm{C} / \mathrm{min}-200^{\circ} \mathrm{C}\left(7{ }^{\circ} \mathrm{C} / \mathrm{min}\right)-250^{\circ} \mathrm{C} \quad(5,86$ $\mathrm{min})-250-20^{\circ} \mathrm{C} / \mathrm{min}-300^{\circ} \mathrm{C} \quad(4,5 \mathrm{~min})$, Injetor: $\left(300^{\circ} \mathrm{C}\right)$. A Figura 3 apresenta os cromatogramas obtidos no modo MS/MS para o Método B e para o Método E. Pode ser observado que o Método E apresentou boa resolução para os três últimos picos e em tempo mais reduzido que o Método B. O Método E foi o selecionado para construção da curva analítica. $\mathrm{O}$ método MS/MS permitiu ganhos em detectabilidade em comparação ao método SCAN.

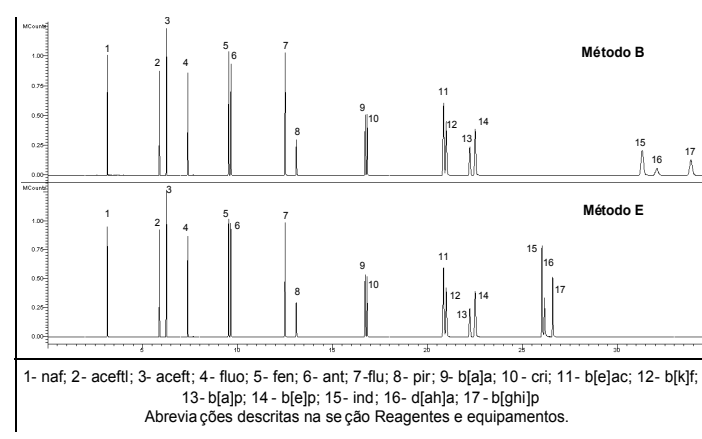

Figure 3. Cromatogramas obtidos na análise de simultânea de 17 HPAs no modo MS/MS, sob as condições de temperatura do Método B e do Método E.

O modo MS/MS oferece vantagens sobre o modo SCAN em relação a selectividade e detectabilidade. No modo MS/MS obtêm-se maior seletividade que o modo SCAN, pois permanecem no trap apenas o fragmento (íon pai) selecionado para refragmentação, seguindo para o detector apenas os íons filho. Dessa maneira, o modo MS/MS é uma ferramenta muito útil na análise de matrizes complexas, que podem possuir outras moléculas que eluem no mesmo tempo de retenção do analito, que no modo SCAN chegariam juntamente com os analitos ao detector, podendo ocasionar erros na identificação e quantificação do analito. Além disso, o modo MS/MS muitas vezes apresenta ganhos em detectabilidade em relação ao modo SCAN, devido a fatores como a obtenção de maior relação sinal/ruído e menor interferência de outros componentes da amostra. O método MS/MS desenvolvido para análise de 17 HPAs apresentou limites de detecção e de quantificação cerca de 5 vezes inferiores que os encontrados para as mesmas moléculas analisadas no modo SCAN, nas mesmas condições cromatográficas.

As curvas analíticas foram construídas a partir da injeção de 9 soluções padrão de 17 
HPAs, em concentrações que variaram de 1 a $0,001 \mu \mathrm{g} \mathrm{mL}^{-1}$. Cada ponto da curva analítica foi injetado cinco vezes. Os limites de detecção e de quantificação observados, coeficientes de correlação das curvas analíticas e o coeficiente de variação médio para cada HPA analisado encontram-se na Tabela 3.

Table 3. Limites de detecção (LD) e limites de quantificação (LQ) para 17 HPAs no sistema GC$\mathrm{MS} / \mathrm{MS}$, em ng $\mathrm{mL}^{-1}$.

\begin{tabular}{ccccc}
\hline HPA & L.D. & L.Q. & $\mathrm{R}^{2}$ & $\begin{array}{c}\text { CV médio } \\
(\%)\end{array}$ \\
\hline naf & 1,96 & 7,85 & 0.9996 & 8 \\
aceftl & 1,95 & 7,81 & 0.9999 & 7 \\
aceft & 1,94 & 7,76 & 0.9999 & 8 \\
fluo & 1,93 & 7,73 & 0.9998 & 6 \\
fen & 2,01 & 8,03 & 0.9998 & 8 \\
antr & 1,98 & 7,91 & 0.9998 & 7 \\
flu & 1,98 & 7,94 & 0.9999 & 8 \\
pir & 1,91 & 7,63 & 0.9999 & 7 \\
b[a]a & 1,97 & 7,88 & 0.9998 & 6 \\
cri & 1,97 & 7,86 & 0.9999 & 7 \\
b[e]ac & 1,95 & 7,80 & 0.9995 & 14 \\
b[k]f & 1,98 & 7,94 & 0.9995 & 12 \\
b[a]p & 1,95 & 7,81 & 0.9998 & 6 \\
b[e]p & 1,95 & 7,79 & 0.9994 & 12 \\
ind & 1,92 & 7,69 & 0.9971 & 15 \\
d[ah]a & 1,94 & 7,77 & 0.9954 & 14 \\
b[ghi]p & 1,95 & 7,79 & 0.99765 & 8 \\
\hline
\end{tabular}

Abreviações descritas na seção Reagentes e equipamentos.

\section{Análise de amostras de material particulado atmosférico}

Os procedimentos de extração de HPAs em material particulado descritos na literatura envolvem, em geral, as seguintes etapas: 1-) extração com solventes (soxhlet, banho de ultrasom, extração assistida por microondas ou com solventes pressurizados -ASE); 2-) concentração do extrato (rota evaporador seguido de leve fluxo de nitrogênio); 3-) dissolução do extrato seco em solvente apropriado; 4-) análise[18, 20, 21, 23, 24].

Muitos trabalhos na literatura utilizam uma mistura de diclorometano e metanol na proporção volumétrica de quatro para um (DCM/MeOH (4:1 v/v)) como solvente de extração[21, 24, 25, 26, 27]. Em um estudo recente os autores obtiveram boa recuperação (67-98\%) para doze HPAs em material particulado atmosférico utilizando extração assistida por microondas e uma mistura isovolumétrica de hexano e acetona[23]. Sob as mesmas condições, os resultados foram comparáveis aos obtidos utilizando diclorometano como solvente extrator. Levando em consideração que o diclorometano é um dos solventes mais utilizados para extração de HPAs em material particulado atmosférico[20, 21, 24, 28], pelo trabalho de Karthikeyan et al. (2006)[23] sugere-se que a mistura HEX/ACT $(1: 1 \mathrm{v} / \mathrm{v})$ é um possível candidato à substituição do diclorometano para extração em banho de ultrasson e Soxhlet. Dessa maneira, neste trabalho foi realizado um estudo preliminar para verificar se pode haver viabilidade de utilização da mistura de solventes HEX/ACT (1:1 v/v) em substituição do solvente $\mathrm{DCM} / \mathrm{MeOH}(4: 1 \mathrm{v} / \mathrm{v})$, utilizando banho de ultra-som, sem perdas significativas em extractibilidade.

Os extratos referentes às metades dos filtros contendo material particulado atmosférico, extraídas com cada mistura de solventes, DCM/MeOH (4:1 v/v) e HEX/ACT (1:1 v/v), foram analisadas por GC-MS/MS. Na análise dos extratos foi possível quantificar os HPAs fenantreno, fluoranteno, pireno, criseno, benzo[e]acefenantrileno, benzo[a]pireno, benzo[e]pireno, indeno[1,2,3-cd]pireno, e benzo[ghi]perileno. A Figura 4 apresenta os cromatogramas obtidos na análise de cada amostra, podendo ser observado que apesar de não ter sido efetuado nenhum clean-up dos extratos das

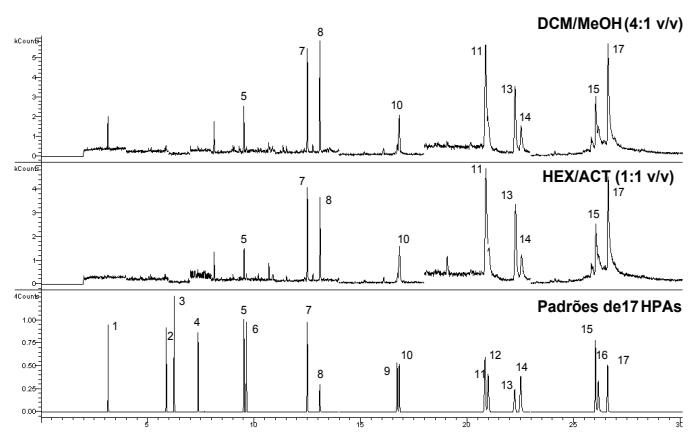

Figure 4. Cromatogramas referentes à injeção de um padrão de 17 HPAs $\left(1 \mu \mathrm{g} \mathrm{mL}{ }^{-1}\right)$ e de extratos de amostras de material particulado atmosférico extraídos com DCM/MeOH (4:1 v/v) e HEX/ACT (1:1 v/v) 
amostras, para reduzir a quantidade de interferentes, foi possível a visualização clara dos picos referentes a cada HPA, demonstrando que o método GC-MS/MS foi seletivamente adequado para análise de amostras de HPAs em material particulado atmosférico.

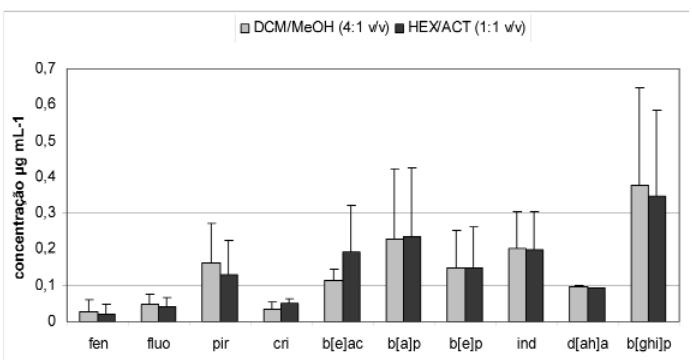

Figure 5. Concentrações médias de HPAs em extratos de amostras de material particulado atmosférico extraídas com DCM/MeOH (4:1 v/v) e com HEX/ACT (1:1 v/v). Abreviações descritas na seção Reagentes e equipamentos.
A Tabela 4 apresenta as concentrações de HPAs obtidas em cada extrato e a Figura 5 apresenta uma comparação da extractibilidade média de cada mistura de solvente para os HPAs presentes na amostra. Observando os dados da Tabela 4 e da Figura 5, pode-se notar que a extractibilidade foi muito semelhante para ambas as misturas de solventes, o que sugere que é possível utilizar a mistura de solvente HEX/ACT $(1: 1 \mathrm{v} / \mathrm{v})$ em substituição à mistura $\mathrm{DCM} / \mathrm{MeOH}$ (4:1 v/v) para extração de HPAs de material particulado atmosférico.

\section{Conclusões}

Foi possível o desenvolvimento de método GC-MS/MS para análise de 17 HPAs considerados como poluentes prioritários pelo NIOSH, obtendo-se ganhos de até 5 vezes em detectabilidade em comparação com o método GC-MS (SCAN). As curvas analíticas apresentaram coe-

Table 4. Concentrações ( $\mu \mathrm{g}$ mL-1) de cada HPA nos extratos de filtros contendo material particulado atmosférico.

\begin{tabular}{ccccccc}
\hline & \multicolumn{2}{c}{ Amostra $1(\mathrm{n}=3)$} & \multicolumn{2}{c}{ Amostra $2(\mathrm{n}=3)$} & \multicolumn{2}{c}{ Amostra 3 (n=3) } \\
\hline HPA & $\begin{array}{c}\text { DCM/MeOH } \\
(4: 1 \mathrm{v} / \mathrm{v})\end{array}$ & $\begin{array}{c}\text { HEX/ACT } \\
(1: 1)\end{array}$ & $\begin{array}{c}\text { DCM/MeOH } \\
(4: 1 \mathrm{v} / \mathrm{v})\end{array}$ & $\begin{array}{c}\text { HEX/ACT } \\
(1: 1 \mathrm{v} / \mathrm{v})\end{array}$ & $\begin{array}{c}\text { DCM/MeOH } \\
(4: 1 \mathrm{v} / \mathrm{v})\end{array}$ & $\begin{array}{c}\text { HEX/ACT } \\
(1: 1 \mathrm{v} / \mathrm{v})\end{array}$ \\
& & & & & & \\
naf & n.d. & n.d. & n.q. & n.d. & n.q. & n.d. \\
aceftl & n.d. & n.d. & n.d. & n.d. & n.d. & n.d. \\
aceft & n.d. & n.d. & n.d. & n.d. & n.d. & n.d. \\
fluo & n.d. & n.d. & n.d. & n.d. & n.d. & n.d. \\
fen & n.q. & n.q. & $0,010 \pm 0,006$ & $0,007 \pm 0,002$ & $0,060 \pm 0,002$ & $0,050 \pm 0,002$ \\
antr & n.d. & n.d. & n.d. & n.d. & n.d. & n.d. \\
flu & $0,021 \pm 0,004$ & $0,018 \pm 0,01$ & $0,035 \pm 0,004$ & $0,029 \pm 0,05$ & $0,080 \pm 0,005$ & $0,070 \pm 0,009$ \\
pir & $0,078 \pm 0,013$ & $0,054 \pm 0,002$ & $0,122 \pm 0,013$ & $0,097 \pm 0,010$ & $0,286 \pm 0,013$ & $0,235 \pm 0,029$ \\
b[a]a & n.d. & n.d. & n.d. & n.d. & n.d. & n.d. \\
cri & $0,042 \pm 0,020$ & $0,037 \pm 0,014$ & $0,046 \pm 0,020$ & $0,047 \pm 0,018$ & $0,064 \pm 0,030$ & $0,065 \pm 0,031$ \\
b[e]ac & $0,134 \pm 0,062$ & $0,111 \pm 0,037$ & $0,127 \pm 0,062$ & $0,127 \pm 0,043$ & $0,330 \pm 0,062$ & $0,339 \pm 0,096$ \\
b[k]f & n.d. & n.d. & n.d. & n.d. & n.d. & n.d. \\
b[a]p & $0,093 \pm 0,046$ & $0,104 \pm 0,024$ & $0,137 \pm 0,046$ & $0,138 \pm 0,033$ & $0,452 \pm 0,034$ & $0,456 \pm 0,045$ \\
b[e]p & $0,097 \pm 0,059$ & $0,089 \pm 0,055$ & $0,087 \pm 0,059$ & $0,079 \pm 0,061$ & $0,265 \pm 0,058$ & $0,279 \pm 0,075$ \\
ind & $0,143 \pm 0,094$ & $0,141 \pm 0,093$ & $0,142 \pm 0,094$ & $0,139 \pm 0,100$ & $0,318 \pm 0,117$ & $0,319 \pm 0,122$ \\
d[ah]a & n.q. & n.q. & n.q. & n.q. & n.q. & n.q. \\
b[ghi]p & $0,207 \pm 0,097$ & $0,202 \pm 0,087$ & $0,227 \pm 0,097$ & $0,214 \pm 0,100$ & $0,691 \pm 0,104$ & $0,622 \pm 0,188$ \\
\hline
\end{tabular}

Abreviações descritas na seção Reagentes e equipamentos.

n: número de injeções no sistema GC-MS/MS

n.d.: não detectado, ou seja, abaixo do limite de detecção, apresentado na Tabela 3

n.q.: não quantificável, ou seja, detectável mas abaixo do limite de quantificação (Tabela 3) 
ficiente de correlação $\geq 0,99$, o que concorda com as recomendações da ANVISA, quanto às exigências de linearidade na validação de métodos analíticos. A repetitividade das áreas foi melhor que $15 \%$, para todos os HPAs em todas as concentrações.

A análise de extratos de amostras de material particulado atmosférico mostrou-se seletiva para os HPAs extraídos dessas amostras. A comparação entre as porcentagens de recuperação obtidas para cada metade de filtro extraídas com DCM/MeOH (4:1 v/v) e HEX/ACT (1:1 v/v) demonstra que é possível a utilização da mistura de solvente HEX/ACT (1:1 v/v) menos tóxica, em substituição à mistura $\mathrm{DCM} / \mathrm{MeOH}(4: 1 \mathrm{v} / \mathrm{v})$, sem perdas significativas em extractibilidade.

Recebido em 06 de junho de 2008

Aceito em 24 de outubro de 2008

J. Cristale, F. S. Silva, M. R. R. Marchi. Development and application of GC-MS/MS method for simultaneous analysis of $17 \mathrm{PAHs}$ in airborne particulate matter.

\begin{abstract}
Polycyclic aromatic hydrocarbons (PAHs) are associated with increased incidence of various types of cancers in humans. These molecules are formed mainly in the incomplete burning of organic matter and can be found in all environmental compartments. North American regulatory agencies for environmental and occupational health areas consider 17 PAHs as air contaminants having priority. This work presents a method for simultaneous analysis of these PAHs using gas chromatography coupled to mass spectrometry, operating in tandem mode (GC-MS/MS). The limits of detection and quantification of this method were 5 times lower than that obtained with GC-MS (SCAN) method. The method was selective in analysis of PAHs from airborne particulate matter. A comparative analysis of two systems of solvents (dichloromethane/methanol $(4: 1 \mathrm{v} / \mathrm{v})$ and hexane/acetone $(1: 1 \mathrm{v} / \mathrm{v}))$ for PAHs extraction, using samples of airborne particulate matter, shows that both mixtures of solvents have similar extraction ability. The results pointed out that it is possible to extract PAHs from airborne particulate matter using a mixture hexane/acetone (1:1), which is less toxic in than dichloromethane/methanol (4:1), usually used in these analyses, without significant losses in the accuracy of the method.
\end{abstract}

Keywords: polycyclic aromatic hydrocarbons; GC-MS/MS; airborne particulate matter.

\section{Referências}

[1] WORLD HEALTH ORGANIZATION. Selected non-heterociclic PAHs: International Programme on Chemical Safety. Geneva, 1998. (Environmental Criteria, 202).

[2] A. D. Pereira Netto, J. C. Moreira, A. E. X. O. Dias, G. Arbilla, 1. F. V. Ferreira, A. S. Oliveira, J. Barek, Quim. Nova 23(6) (2000) 765.

[3] L. Nizzetto, L. Rohmann, R. Gioia, A. Jahnke, C. Temme, J. Dachs, P. Herckes, A. Di Guardo, K. Jones, Environ. Sci. Technol. 42(5) (2008) 1580.

[4] Y. G. Zhu, S. Khan, L. Aijun, S. Zhang, Q. Hu, J, Hazard. Mater. 152 (2008) 506.

[5] S. Tao, Y. H. Cui, F. L. Xu, B. G. Li, J. Cao, W. X. Liu, G. Schmitt, X. J. Wang, W. R. Shen, B. P. Qing, R. Sun, Sci. Total Environ. 320 (2004) 11.

[6] V. Librando, O. Hutzinger, G. Tringali, M. Aresta, Chemosphere 54(8) (2004)1189.
[7] W. Wilcke, W. Amelung, M. Krauss, C. Martius, A. Bandeira, M. Garcia, Org. Geochem. 34 (2003) 1405.

[8] Z. Zhang, J. Huang, G. Yu, H. Hong, Environ. Pollut. 130 (2004) 249.

[9] J. Angerer, C. Mannuschreck, J. Gündel, Int. Arch. Occup. Environ. Health 70 (1997) 365.

[10]S. Zhang, Q. Zhang, S. Darisaw, O. Ehie, G. Wang, Chemosphere 66 (2007) 1057.

[11] P. S. Khillare, T. Agarwal, V. Shridhar, Environ. Monit. Assess. 123 (2006) 151.

[12] EPA. Carcinogen Assessment Group. Evaluation and estimation of potential carcinogenic risks of polynuclear aromatic hydrocarbons. Cincinnati, 1998.

[13] NIOSH. Polynuclear aromatic hydrocarbons by HPLC: method 5506. NIOSH manual of analytical methods (NMAM). 4th ed. 1998.

[14] M. B. Yunker, R. W. Macdonald, R. Vingarzan, H. M. Reginald, D. Goyette, S. Sylvestre, Org. Geochem 33 (2002) 489. 
[15] A. Albinet, E. Leoz-Garziandia, H. Budzinski, E. ViIlenave, Sci. Total Environ 384(1-3) (2007) 280.

[16] J.Cheng, T. Yuan, Q. Wu, W. Zhao, H. Xie, Y. Ma, J. Ma, W. Wang, Water, Air, Soil Pollut. 183 (2007) 437.

[17] L. Z. Zhu, J. Wang, J. Environ. Sci. China 17(3) (2005) 365. [18] C. Y. M. SANTOS, D. A. AZEVEDO, F. R. AQUINO NETO, Atmos. Environ. 36 (2002) 3009.

[19] N. Re-Poppi, G. L. Stroher, J. L. Raposo, J. B. G. Souza, Microchem. J. 86 (2007) 112.

[20] T. Ohura, T. Amagai, T. Sugiyama, M. Fusaya, H. Matsushita, Atmos. Environ. 38 (2004) 2045.

[21] C. Minoia, S. Magnaghi, G. Micoli, M. L. Fiorentino, R. Turci, S. Angeleri, A. Berri, Sci. Total Environ 198(1) (1997) 33. [22] Varian Chromatography Systems. 1996. Saturn 2000 GC/MS: Advanced MS Techniques. U.S.A.: Varian Associates, Inc.
[23] S. Karthikeyan, R. Balasubramanian, S. W. See, Talanta 69 (2006) 79.

[24] A. F .L. Godoi, K. Ravindra, R. H. M. Godoi, S. J. Andrade, M. Santiago-Silva, L. V. Vaeck, R. Van Grieken, J. Chromatogr., A 1027 (2004) 49.

[25] N. Ré-Poppi, M. R. Santiago-Silva, Chromatographia 55 (2002) 475.

[26] N. Re-Poppi, M. Santiago-Silva, Atmos. Environ. 39 (2005) 2839.

[27] ANDRADE, S. J. 2004.. Tese (Doutorado em Química) Instituto de Química, Universidade Estadual Paulista, Araraquara, (2004) 120.

[28] S. Kumar, A. Bhargava, R. N. Khanna, S. K. Bhargava, Atmos. Environ. 38 (2004) 4761. 